University of Wollongong

Research Online

Australian Institute for Innovative Materials -

Papers

Australian Institute for Innovative Materials

$1-1-2017$

Efficient visible-light photocatalysts by constructing dispersive energy band with anisotropic $p$ and s-p hybridization states

\author{
Haifeng Feng \\ University of Wollongong, hf533@uowmail.edu.au \\ Yi Du \\ University of Wollongong, ydu@uow.edu.au \\ Cong Wang \\ Beihang University \\ Weichang Hao \\ Beijing University, University of Wollongong, whao@buaa.edu.cn
}

Follow this and additional works at: https://ro.uow.edu.au/aiimpapers

Part of the Engineering Commons, and the Physical Sciences and Mathematics Commons

Research Online is the open access institutional repository for the University of Wollongong. For further information contact the UOW Library: research-pubs@uow.edu.au 


\title{
Efficient visible-light photocatalysts by constructing dispersive energy band with anisotropic $p$ and s-p hybridization states
}

\author{
Abstract \\ Highly efficient visible-light photocatalysts are strongly in demand because they make it possible to \\ achieve higher efficiency in utilization of solar energy, w hich is renewable and abundant. A narrow band \\ gap, effective separation of photoexcited charge carriers, and proper band-edge positions, which depend \\ on the band structure of the photocatalyst, are crucial for solar-energy conversion efficiency. In this \\ review, we have summarized reports on the strategy of exploiting highly efficient visible-light \\ photocatalysts by constructing their band structure with anisotropic $p$ and $s-p$ hybridization states. Taking \\ advantage of their dispersive and easily tunable band properties, enhanced efficiency in solar light \\ absorption, efficient separation of photoexcited charge carriers and catalytic reactions are expected to be \\ simultaneously achieved.

\section{Disciplines} \\ Engineering | Physical Sciences and Mathematics

\section{Publication Details} \\ Feng, H., Du, Y., Wang, C. \& Hao, W. (2017). Efficient visible-light photocatalysts by constructing dispersive \\ energy band with anisotropic $p$ and s-p hybridization states. Current Opinion in Green and Sustainable \\ Chemistry, 6 93-100.
}




\title{
Efficient visible-light photocatalysts by constructing dispersive energy band with anisotropic $p$ and $s-p$ hybridization states
}

\author{
Haifeng Feng ${ }^{1,2,3}$, Yi Du ${ }^{1,2,3}$, Cong Wang ${ }^{1}$, Weichang $\mathrm{Hao}^{1,2,3^{*}}$ \\ ${ }^{1}$ Department of Physics and Key Laboratory of Micro-Nano Measurement, Manipulation and \\ Physics, Ministry of Education, Beihang University, Beijing 100191, China. \\ ${ }^{2}$ Institute for Superconducting and Electronic Materials, University of Wollongong, NSW \\ 2500, Australia. \\ ${ }^{3}$ BUAA-UOW Joint Research Centre, Beihang University, Beijing 100191, China. \\ * To whom correspondence should be addressed. Email: whao@buaa.edu.cn.
}

\begin{abstract}
Highly efficient visible-light photocatalysts are strongly in demand because they make it possible to achieve higher efficiency in utilization of solar energy, which is renewable and abundant. A narrow band gap, effective separation of photoexcited charge carriers, and proper band-edge positions, which depend on the band structure of the photocatalyst, are crucial for solar-energy conversion efficiency. In this review, we have summarized reports on the strategy of exploiting highly efficient visible-light photocatalysts by constructing their band structure with anisotropic $p$ and $s-p$ hybridization states. Taking advantage of their dispersive and easily tunable band properties, enhanced efficiency in solar light absorption, efficient separation of photoexcited charge carriers and catalytic reactions are expected to be simultaneously achieved.
\end{abstract}

Keywords: $p$ state, $s-p$ hybridization, anisotropic orbitals, visible-light photocatalysts, solar energy. 


\section{Main text}

\section{Introduction}

In recent decades, the excessive use of fossil fuels has resulted in a serious global crisis involving energy shortages and environmental pollution. There is a great demand to develop green and sustainable technologies for clean energy production and environmental remediation. Photocatalysis, through which one can convert solar energy into chemical energy, has been regarded as one of the most promising strategies. It has been demonstrated to exhibit great potential in several applications, including photocatalytic water splitting, photosynthesis, and the treatment of pollutants in aqueous or gas phase [1-4]. Unfortunately, reliable photocatalysts are scarce, although the appeal of the direct conversion of solar into chemical energy via semiconductor compounds has been recognized for a long time. This is mainly due to their low solar-energy conversion efficiency, especially in the visible-light spectrum.

Generally, a typical photocatalytic process involves three steps: (i) absorption of solar light (with the efficiency denoted as $\eta_{\mathrm{A}}$ ), (ii) separation of photoexcited charge carriers (with the efficiency denoted as $\eta_{\mathrm{s}}$ ), and (iii) reduction or oxidation reactions at the surface to complete the solar energy conversion (with the efficiency denoted as $\eta_{\mathrm{C}}$ ). The overall efficiency $(\eta)$ is therefore determined by multiplying the efficiencies of the individual steps: $\eta=$ $\eta_{\mathrm{A}} \times \eta_{\mathrm{S}} \times \eta_{\mathrm{C}}$. Thus, several criteria are essential for the design and development of novel photocatalysts that possess high solar-energy conversion efficiency. 
First of all, the band gaps of the photocatalysts should be narrow enough $\left(E_{\mathrm{g}}<\right.$ 3.0 eV), which allow absorption of both ultraviolet (UV) and visible light in the solar spectrum. This is because UV and visible light account for 5\% and $43 \%$ of the solar spectrum, respectively. Secondly, a high separation rate and high mobility of the photoexcited charge carriers (electrons and holes) are essential for high-efficiency photocatalysts. The former can lead to high quantum conversion efficiency, while the latter increases the effective charge-carrier diffusion length and thus enhances the photocatalytic activity. For semiconductors, the mobility of electrons and holes is determined by their effective mass $\left(m^{*}\right)$. As shown in Eq. (1), the $m^{*}$ is estimated to the second order derivative of energy $(E)$ with respect to the wave vector $(k)$, which is reflected by the curvature of the band edges.

$$
\frac{1}{m^{*}}=\frac{1}{\hbar^{2}} \times \frac{d^{2} E}{d k^{2}}
$$

As illustrated in Figure 1a, a larger curvature of the band leads to a small $m^{*}$ (light effective mass) of the charge carriers. This means the dispersion of the electronic band determines $m^{*}$, that is, the more dispersive the band the smaller $m^{*}$ will be, and consequently, the higher the mobility it will have. In contrast, a dispersion-less band leads to large $m^{*}$ (heavy effective mass) of charge carriers with low mobility. Thirdly, the positions of the valence and conduction bands (VB and $\mathrm{CB}$ ) are of importance to determine the chemical potential of photoexcited electrons and holes, which have significant impact on the efficiency of different photocatalytic reactions (Figure 1b). The overall 
solar-energy conversion during the photocatalysis process is, therefore, determined by the electronic structures of photocatalysts.

Recently, semiconductor photocatalysts mainly or partially constructed by p-block elements have been reported to exhibit high visible-light photocatalytic activity and considerable efficiency [5-11]. Their alluring visible-light photocatalytic performances could be ascribed to their unique electronic structures, which involve $p$ orbitals or $s-p$ hybridized orbitals in constructing their band edges. Through $p$ or $s$ - $p$ hybridizations, $p$ electrons from $p$-block elements lift the top of the $\mathrm{VB}$ and/or lower the bottom of the $\mathrm{CB}$ in photocatalysts, hence reducing the band gap [12-15]. In addition, spatially anisotropic $p$ and $s-p$ hybridization states create highly dispersive band structures, which promote separation and mobility of photoexcited electron-hole pairs by reducing their effective mass. All these features significantly contribute to high energy conversion efficiency under solar light. Thus, through selecting suitable elements, which have valence electrons residing in $s$ or $p$ orbitals, it is expected that we could design and develop visible-light photocatalysts with the desired band structure. This strategy is essentially in accordance with the fact that almost all the superior electronic devices in the semiconductor industry are based on $p$-block elemental semiconductors or compounds with $s-p$ hybridizations, such as $\mathrm{Si}, \mathrm{Ge}$, and GaAs. One of their major advantages over other semiconductors is the high mobility of their charge carriers, which originates from their dispersive $p$ or $s-p$ hybridized bands. Based on the above 
principles, compounds constructed from the elements in three regions (IA and IIA, IB and IIB, and the $p$-block) in the periodic table have aroused our interest as shown in Figure 1c. This is because their configurations of $d$ orbitals are either $d^{0}$ or $d^{10}$ in these compounds, which has the result that their band edges are constructed from anisotropic $p$ or $s-p$ hybridization states. Therefore, taking advantage of the easily tunable band gap and anisotropic orbitals, exploiting semiconductors built from these elements has great potential to create visible-light photocatalysts with high overall efficiency. This review will exclusively focus on the recent advances in visible-light photocatalysts with electronic structures constructed from anisotropic $p$ or $s-p$ hybridization states.

\section{Visible light photocatalysts composed of only $p$ elements}

A typical example of the visible-light photocatalysts that are only constructed from $p$-block elements, is the bismuth oxyhalides family, $\mathrm{Bi}_{x} \mathrm{O}_{y} \mathrm{X}_{z}$ $(\mathrm{X}=\mathrm{Cl}, \mathrm{Br}$, and $\mathrm{I})$. As a heavy metal, bismuth atoms tend to form very weak bonds with non-metal elements, which generally tend to have small molecular orbital overlap and favours the establishment of narrow band gaps. Along with a narrow band gap, a small effective mass may to be acquired for semiconductors with similar crystal and band structures, according to the $\boldsymbol{k} \cdot \boldsymbol{p}$ perturbation theory. Various $\mathrm{Bi}_{x} \mathrm{O}_{y} \mathrm{X}_{z}$ compounds exhibit high visible-light activity towards photocatalytic degradation of organic and inorganic toxic substances, water splitting, and $\mathrm{N}_{2}$ fixation [5,6,16-25]. Their VB maximum (VBM) is mainly composed of $\mathrm{O} 2 p$ and $\mathrm{X} n p$ states $(n=3,4$, and 5 for $\mathrm{Cl}, \mathrm{Br}$, and $\mathrm{I}$, 
respectively). Their $\mathrm{CB}$ minimum $(\mathrm{CBM})$ in most cases is constructed from $\mathrm{Bi}$ $6 p$ states [26-28]. Thus, the band structures of $\mathrm{Bi}_{x} \mathrm{O}_{y} \mathrm{X}_{z}$ are expected to be tunable via the halogen species and the ratios of $\mathrm{Bi}$ : $\mathrm{O}: \mathrm{X}$. Meanwhile, due to the dispersive properties of the $p$ and $s-p$ hybridization states, high-mobility charge carriers can be obtained in many $\mathrm{Bi}_{x} \mathrm{O}_{y} \mathrm{X}_{z}$ compounds. As shown in Figure $2 \mathrm{a}$ and $\mathrm{b}$, both experimental and theoretical works have suggested that the band gap of $\mathrm{BiOX}$ can be gradually narrowed from $3.4 \mathrm{eV}$ for $\mathrm{BiOCl}$ to 2.8 $\mathrm{eV}$ for $\mathrm{BiOBr}$ and $1.9 \mathrm{eV}$ for $\mathrm{BiOI}$, due to the increasing participation of the $\mathrm{X}$ $n p$ states $[29,30]$. As a result, Zhang et al. proved that BiOI shows a wider visible-light absorption range than $\mathrm{BiOCl}$ and $\mathrm{BiOBr}$, and higher visible-light photocatalytic activity towards degradation of methyl orange (MO) [31]. On the other hand, due to the appropriate $\mathrm{VB}$ edge, $\mathrm{BiOBr}$ and $\mathrm{BiOCl}$ exhibited much higher oxygen evolution efficiency and better photocatalytic degradation of Rhodamine $\mathrm{B}(\mathrm{RhB})$ and phenol under visible light or simulated sunlight irradiation than BiOI $[29,32]$. As shown in Figure $2 \mathrm{~d}$ and e, Bhachu et al. demonstrated that $\mathrm{BiOBr}$ also had much superior water oxidation activity compared to $\mathrm{BiOI}$ and $\mathrm{BiOCl}$ under simulated sunlight irradiation, due to its more positive VB edge [33].

Adjusting the ratio of $\mathrm{Bi}: \mathrm{O}: \mathrm{X}$ in $\mathrm{Bi}_{x} \mathrm{O}_{y} \mathrm{X}_{z}$ has also been demonstrated to be an effective approach to obtain visible-light photocatalysts with the desired electronic structure [6,34-36]. Shang et al. reported that $\mathrm{Bi}_{24} \mathrm{O}_{31} \mathrm{Br}_{10}\left(E_{\mathrm{g}} \approx 2.8 \mathrm{eV}\right.$, similar to $\mathrm{BiOBr}$ ) exhibited considerable photocatalytic activity towards $\mathrm{Cr}(\mathrm{VI})$ 
ion reduction and $\mathrm{H}_{2}$ evolution through water splitting (Figure $3 \mathrm{~b}$ and c) [6]. $\mathrm{BiOBr}$ cannot split water to release $\mathrm{H}_{2}$ through photocatalytic reactions, however, because its CBM is more positive than the electrode potential of $\mathrm{H}^{+} / \mathrm{H}_{2}$. While in the case of $\mathrm{Bi}_{24} \mathrm{O}_{31} \mathrm{Br}_{10}$, as illustrated in Figure 3a, the CBM, mainly consisting of hybridized $\mathrm{Bi} 6 p$ and $\mathrm{Br} 4 s$ orbitals, is uplifted to be more negative than the electrode potential of $\mathrm{H}^{+} / \mathrm{H}_{2}$, which enables $\mathrm{Bi}_{24} \mathrm{O}_{31} \mathrm{Br}_{10}$ to reduce water into $\mathrm{H}_{2}$ under visible-light irradiation. In addition, the hybridization $s-p$ orbitals lead to a dispersive band, which is expected to increase its efficiency through promoting the separation of the photoexcited electrons and holes.

It should be noted that for $\mathrm{Bi}_{x} \mathrm{O}_{y} \mathrm{X}_{z}$ photocatalysts, the nature of their internal electric field contributes to the efficiency of separating photoexcited charge carriers, which is induced by their layered structure, consisting of interleaving positive [Bi-O] layers and negative $\mathrm{X}^{-}$layers $[18,33,37,38]$. In a recent work, $\mathrm{Li}$ et al. demonstrated that substituting $\mathrm{C}$ atoms on $\mathrm{Cl}$ sites in $\mathrm{Bi}_{3} \mathrm{O}_{4} \mathrm{Cl}$ is an efficient way to enhance the internal electric field, which dramatically increases its bulk-charge separation efficiency to as high as $80 \%$. The enhanced separation efficiency of photoexcited electrons and holes led to a higher capability for photocatalytic water oxidation under visible light without any noble metals and electron scavengers [39].

Besides $\mathrm{Bi}_{x} \mathrm{O}_{y} \mathrm{X}_{z}$, some new $p$-block compounds have emerged as highly active visible-light photocatalysts due to their appropriate band structures. Huang et al. reported a ternary boron carbon nitride $(\mathrm{B}-\mathrm{C}-\mathrm{N})$ alloy, which 
showed great potential in visible-light photocatalytic water splitting and $\mathrm{CO}_{2}$ reduction due to its adjustable band gap and two-dimensional (2D) electron system [40]. $P$-block elemental photocatalysts, such as Si [41], S [42], and P [43] have also been reported to show visible-light photocatalytic activity because their electronic structures are tunable towards crystalline structures and phases. With the special advantages of 2D materials, several monolayer or few-layer materials in composites with $p$-block elements are expected to exhibit high visible-light photocatalytic activity due to their narrow band gaps and special electronic structures. For examples, black phosphorus (BP) is a narrow bandgap semiconductor with a flexible and adjustable band-gap value. In addition, its CBM is highly asymmetric, and there is anisotropic charge mobility between the electrons and the holes. BP is thus predicted to be an excellent candidate for photocatalysis [44]. Monolayer red phosphorus (RP) is also predicted to be a semiconductor with a direct band gap of $2.4 \mathrm{eV}$, and to possess a very dispersive conduction band, which will be beneficial to the separation of photoexcited charge carriers [45].

3. Visible light photocatalysts integrating $p$-block elements with other elements with $d^{0}$ or $d^{10}$ electron configurations

Integrating $p$-block electron configuration states with other elements with $d^{0}$ or $d^{10}$ electron configurations in photocatalysts is another effective way to obtain charge carriers with small effective mass and high mobility, because dispersive $s-p$ hybridization states are easily formed without the participation of 
$d$ orbitals. $\mathrm{Ag}_{10} \mathrm{Si}_{4} \mathrm{O}_{13}$, one of the silver silicates, is reported to be an excellent visible-light photocatalyst towards the degradation of organic compounds, owing to its unique band structure and ferroelectric polarization [46-50]. As shown in Figure 4a, the hybridized CBMs are very dispersive, whereas the VBMs are quite flat. The photoexcited electrons, consequently, have a small effective mass, which enables photoexcited electrons to easily move to surface active sites due to their high mobility. In contrast, heavy holes will be generated in the photoexcitation process. This special electron configuration is beneficial to the separation of photoexcited charge carriers and promotes solar-energy conversion efficiency in photocatalysis. Lou et al. found that the visible light absorption and band structures of amorphous silver silicates are tunable (Figure $4 \mathrm{~b}$ and $\mathrm{c}$ ) by the $\mathrm{Ag} / \mathrm{Si}$ ratio. With an $\mathrm{Ag} / \mathrm{Si}$ ratio of 3.2, silver silicate exhibits optimal photocatalytic activity due to its suitable band gap and high redox activity of carriers [51].

$\mathrm{M}\left(\mathrm{BiO}_{3}\right)_{\mathrm{n}}$, where $\mathrm{M}$ are alkali metals, including $\mathrm{Na}, \mathrm{Li}, \mathrm{K}, \mathrm{Ca}$, and $\mathrm{Mg}$ [52-54], is another family of visible-light photocatalysts with high photocatalytic oxidation activity towards the decomposition of organic pollutants. With the empty $6 s$ orbital of $\mathrm{Bi}^{5+}$, the $s$ states of $\mathrm{Bi}$ and the alkali metals have the opportunity to participate in both the CBM and the VBM, forming the $s-p$ hybridization states with strong dispersion. For example, the $\mathrm{VBM}$ of $\mathrm{NaBiO}_{3}$ is constructed mainly from $\mathrm{O} 2 p$ orbitals, while the $\mathrm{CBM}$ is constructed from the hybridization of $\mathrm{Na} 3 s$ and $\mathrm{O} 2 p$ orbitals, which produces light electrons, and 
thus contributes to the high activity of this material.

\section{Conclusion}

In this review, recent developments are reviewed in exploiting highly efficient visible-light photocatalysts through constructing their electronic structure with $p$ and $s-p$ hybridization states. Some photocatalysts have already been demonstrated to exhibit excellent visible-light photocatalytic activity, especially towards photocatalytic oxidation, by taking advantage of their tunable band structure and dispersive band features. It should be noted that substantial increases in the efficiency of visible-light photocatalysts are still urgently needed to realize wide industrial applications of photocatalysis. To achieve this goal, an in-deep understanding of each step of the photocatalytic processes is essential to acquire a high overall solar-energy conversion efficiency. Considering that there is an appreciable number of semiconductors that have not been exploited through quantitatively integrating the elements in the regions that we marked in Figure 1c, visible-light photocatalysts with a substantially increased efficiency have a high probability of being captured by this strategy.

\section{Acknowledgements}

The authors are grateful for financial support from the National Natural

Science Foundation of China (51272015, 51472016, and 51672018), the Fundamental Research Funds for the Central Universities (YWF-16-JCTD-B-03 
and YWF-16--BJ-J-44). H.F.F. and Y.D. thank the Australian Research Council (ARC) for partial support of this work through a Discovery Project (DP140102581, DP170101467). The authors thank Dr T. Silver for her valuable comments on this work.

\section{References}

Papers of particular interest, published within the period of review, have been highlighted as: $*$ of special interest.

[1] Q. Wang, T. Hisatomi, Q. Jia, H. Tokudome, M. Zhong, C. Wang, Z. Pan, T. Takata, M. Nakabayashi, N. Shibata, Y. Li, I.D. Sharp, A. Kudo, T. Yamada, K. Domen, Scalable water splitting on particulate photocatalyst sheets with a solar-to-hydrogen energy conversion efficiency exceeding $1 \%$, Nat. Mater. 15 (2016) 611-615.

[2] J. Liu, M.S. Kelley, W. Wu, A. Banerjee, A.P. Douvalis, J. Wu, Y. Zhang, G.C. Schatz, M.G. Kanatzidis, Nitrogenase-mimic iron-containing chalcogels for photochemical reduction of dinitrogen to ammonia, Proc. Natl. Acad. Sci. USA 113 (2016) 5530-5535.

[3] H. Park, H.-H. Ou, U. Kang, J. Choi, M.R. Hoffmann, Photocatalytic conversion of carbon dioxide to methane on $\mathrm{TiO}_{2} / \mathrm{CdS}$ in aqueous isopropanol solution, Catal. Today 266 (2016) 153-159.

[4] Y. Li, S. Ouyang, H. Xu, X. Wang, Y. Bi, Y. Zhang, J. Ye, Constructing Solid-gas-interfacial fenton reaction over alkalinized- $\mathrm{C}_{3} \mathrm{~N}_{4}$ photocatalyst to 
achieve apparent quantum yield of $49 \%$ at $420 \mathrm{~nm}$, J. Am. Chem. Soc. 138 (2016) 13289-13297.

[5] H. Li, J. Shang, Z. Ai, L. Zhang, Efficient visible light nitrogen fixation with $\mathrm{BiOBr}$ nanosheets of oxygen vacancies on the exposed $\{001\}$ facets, J. Am. Chem. Soc. 137 (2015) 6393-6399.

[6] J. Shang, W. Hao, X. Lv, T. Wang, X. Wang, Y. Du, S.X. Dou, T. Xie, D. Wang, J. Wang, Bismuth oxybromide with reasonable photocatalytic reduction activity under visible light, ACS Catal. 4 (2014) 954-961.

[7] H. Fujito, H. Kunioku, D. Kato, H. Suzuki, M. Higashi, H. Kageyama, R. Abe, Layered perovskite oxychloride $\mathrm{Bi}_{4} \mathrm{NbO}_{8} \mathrm{Cl}$ : A stable visible light responsive photocatalyst for water splitting, J. Am. Chem. Soc. 138 (2016) 2082-2085 $\left(* \mathrm{Bi}_{4} \mathrm{NbO}_{8} \mathrm{Cl}\right.$ is an efficient visible-light photocatalyst towards $\mathrm{O}_{2}$-evolving due to its highly dispersive $\mathrm{O}-2 p$ orbitals).

[8] J. Liu, Y. Liu, N. Liu, Y. Han, X. Zhang, H. Huang, Y. Lifshitz, S.-T. Lee, J. Zhong, Z. Kang, Metal-free efficient photocatalyst for stable visible water splitting via a two-electron pathway, Science 347 (2015) 970-974.

[9] Y. Kang, Y. Yang, L.-C. Yin, X. Kang, G. Liu, H.-M. Cheng, An amorphous carbon nitride photocatalyst with greatly extended visible-light-responsive range for photocatalytic hydrogen generation, 27 (2015) 4572-4577.

[10] D.J. Martin, G. Liu, S.J.A. Moniz, Y. Bi, A.M. Beale, J. Ye, J. Tang, Efficient visible driven photocatalyst, silver phosphate: performance, understanding and perspective, Chem. Soc. Rev. 44 (2015) 7808-7828. 
[11] L. Yue, S. Wang, G. Shan, W. Wu, L. Qiang, L. Zhu, Novel MWNTs$\mathrm{Bi}_{2} \mathrm{WO}_{6}$ composites with enhanced simulated solar photoactivity toward adsorbed and free tetracycline in water, Appl. Catal. B 176-177 (2015) 11-19.

[12] T. Takata, C. Pan, K. Domen, Recent progress in oxynitride photocatalysts for visible-light-driven water splitting, Sci. Technol. Adv. Mater. 16 (2015) 033506.

[13] J. Boltersdorf, I. Sullivan, T.L. Shelton, Z. Wu, M. Gray, B. Zoellner, F.E. Osterloh, P. A. Maggard, Flux synthesis, optical and photocatalytic properties of n-type $\mathrm{Sn}_{2} \mathrm{TiO}_{4}$ : hydrogen and oxygen evolution under visible light, Chem. Mater. 28 (2016) 8876-8889.

[14] Y. Huang, W. Fan, B. Long, H. Li, F. Zhao, Z. Liu, Y. Tong, H. Ji, Visible light $\mathrm{Bi}_{2} \mathrm{~S}_{3} / \mathrm{Bi}_{2} \mathrm{O}_{3} / \mathrm{Bi}_{2} \mathrm{O}_{2} \mathrm{CO}_{3}$ photocatalyst for effective degradation of organic pollutions, Appl. Catal. B 185 (2016) 68-76.

[15] T. Takata, C. Pan, K. Domen, Design and development of oxynitride photocatalysts for overall water splitting under visible light irradiation, ChemElectroChem 3 (2016) 31-37.

[16] J. Di, J. Xia, M. Ji, S. Yin, H. Li , H. Xu, Q. Zhang, H. Li, Controllable synthesis of $\mathrm{Bi}_{4} \mathrm{O}_{5} \mathrm{Br}_{2}$ ultrathin nanosheets for photocatalytic removal of ciprofloxacin and mechanism insight, J. Mater. Chem. A 3 (2015) $15108-15118$.

[17] W.W. Lee, C.-S. Lu, C.-W. Chuang, Y.-J. Chen, J.-Y. Fu, C.-W. Siao, C.-C. 
Chen, Synthesis of bismuth oxyiodides and their composites:

Characterization, photocatalytic activity, and degradation mechanisms, RSC Adv. 5 (2015) 23450-23463.

[18] H. Feng, Z. Xu, L. Wang, Y. Yu, D. Mitchell, D. Cui, X. Xu, J. Shi, T. Sannomiya, Y. Du, W. Hao, S.X. Dou, Modulation of photocatalytic properties by strain in 2D BiOBr nanosheets, ACS Appl. Mater. Interfaces 7 (2015) 27592-27596.

[19] Z.-Y. Zhao, W.-W. Dai, Electronic structure and optical properties of BiOI ultrathin films for photocatalytic water splitting, Inorg. Chem. 54 (2015) $10732-10737$.

[20] J. Han, G. Zhu, M. Hojamberdiev, J. Peng, X. Zhang, Y. Liu, B. Ge, P. Liu, Rapid adsorption and photocatalytic activity for Rhodamine B and Cr(VI) by ultrathin BiOI nanosheets with highly exposed $\{001\}$ facets, New J. Chem. 39 (2015) 1874-1882.

[21] C.-Y. Wang, X. Zhang, X.-N. Song, W.-K. Wang, H.-Q. Yu, Novel $\mathrm{Bi}_{12} \mathrm{O}_{15} \mathrm{Cl}_{6}$ photocatalyst for the degradation of bisphenol A under visible-light irradiation, ACS Appl. Mater. Interfaces 8 (2016) 5320-5326 (* A novel $p$-block photocatalyst, $\mathrm{Bi}_{12} \mathrm{O}_{15} \mathrm{Cl}_{6}$, is demonstrated to be a good candidate for the degradation of diverse pollutants in water and wastewater under solar light).

[22] Z.H. Ai, W.K. Ho, S.C. Lee, L.Z. Zhang, Efficient photocatalytic removal of $\mathrm{NO}$ in indoor air with hierarchical bismuth oxybromide nanoplate 
microspheres under visible light, Environ. Sci. Technol. 43 (2009) 41434150.

[23] L. Wang, J. Shang, W. Hao, S. Jiang, S. Huang, T. Wang, Z. Sun, Y. Du, S.X. Dou, T. Xie, D. Wang, J. Wang, A dye-sensitized visible light photocatalyst- $\mathrm{Bi}_{24} \mathrm{O}_{31} \mathrm{Cl}_{10}$, Sci. Rep. 4 (2014) 7384.

[24] W. Hao, Y. Gao, X. Jing, W. Zou, Y. Chen, T. Wang, Visible light photocatalytic properties of metastable $\gamma-\mathrm{Bi}_{2} \mathrm{O}_{3}$ with different morphologies, J. Mater. Sci. Technol. 30 (2014) 192-196.

[25] W. Zou, W.-C. Hao, X. Xin, T.-M. Wang, Visible-light photocatalytic degradation of $\mathrm{RhB}$ by $\mathrm{Bi}_{2} \mathrm{O}_{3}$ polymorphs, Chinese J. Inorg. Chem. 25 (2009) 1971-1976.

[26] X. Liu, Y. Su, Q. Zhao, C. Du, Z. Liu, Constructing $\mathrm{Bi}_{24} \mathrm{O}_{31} \mathrm{Cl}_{10} / \mathrm{BiOCl}$ heterojunction via a simple thermal annealing route for achieving enhanced photocatalytic activity and selectivity, Sci. Rep. 6 (2016) 28689.

[27] S. Jiang, L. Wang, W. Hao, W. Li, H. Xin, W. Wang, T. Wang, Visible-light photocatalytic activity of S-doped $\alpha-\mathrm{Bi}_{2} \mathrm{O}_{3}$, J. Phys. Chem. C 119 (2015) $14094-14101$.

[28] Z. Xu, W. Hao, Q. Zhang, Z. Fu, H. Feng, Y. Du, S.X. Dou, Indirect-direct band transformation of few-layer BiOCl under biaxial strain, J. Phys. Chem. C 120 (2016) 8589-8594.

[29] H. An, Y. Du, T. Wang, C. Wang, W. Hao, J. Zhang, Photocatalytic properties of $\mathrm{BiOX}(\mathrm{X}=\mathrm{Cl}, \mathrm{Br}$, and I), Rare Metals 27 (2008) 243-250. 
[30] A.M. Ganose, M. Cuff, K.T. Butler, A. Walsh, D.O. Scanlon, Interplay of orbital and relativistic effects in bismuth oxyhalides: $\mathrm{BiOF}, \mathrm{BiOCl}, \mathrm{BiOBr}$, and BiOI, Chem. Mater. 28 (2016) 1980-1984 (* Revealing the role of the halide anion in determining the electronic structures of $\mathrm{BiOX}$ ).

[31] X. Zhang, Z. Ai, F. Jia, L. Zhang, Generalized one-pot synthesis, characterization, and photocatalytic activity of hierarchical $\mathrm{BiOX}(\mathrm{X}=\mathrm{Cl}$, Br, I) nanoplate microspheres, J. Phys. Chem. C 112 (2008) 747-753.

[32] H. Huang, X. Han, X. Li, S. Wang, P.K. Chu, Y. Zhang, Fabrication of multiple heterojunctions with tunable visible light-active photocatalytic reactivity in $\mathrm{BiOBr}-\mathrm{BiOI}$ full-range composites based on microstructure modulation and band structures, ACS Appl. Mater. Interfaces 7 (2015) $482-492$.

[33] D.S. Bhachu, S.J.A. Moniz, S. Sathasivam, D.O. Scanlon, A. Walsh, S.M. Bawaked, M. Mokhtar, A.Y. Obaid, I.P. Parkin, J. Tang, C.J. Carmalt, Bismuth oxyhalides: Synthesis, structure and photoelectrochemical activity, Chem. Sci. 7 (2016) 4832-4841. (* In BiOX, the oxidation activity of water under simulated sunlight irradiation is determined by its electronic structures, especially the position of VB).

[34] Y. Su, C. Ding, Y. Dang, H. Wang, L. Ye, X. Jin, H. Xie, C. Liu, First hydrothermal synthesis of $\mathrm{Bi}_{5} \mathrm{O}_{7} \mathrm{Br}$ and its photocatalytic properties for molecular oxygen activation and RhB degradation, Appl. Surf. Sci. 346 (2015) 311-316. 
[35] Y. Bai, T. Chen, P. Wang, L. Wang, L. Ye, Bismuth-rich $\mathrm{Bi}_{4} \mathrm{O}_{5} \mathrm{X}_{2}(\mathrm{X}=\mathrm{Br}$, and I) nanosheets with dominant $\{101\}$ facets exposure for photocatalytic $\mathrm{H}_{2}$ evolution, Chem. Eng. J. 304 (2016) 454-460.

[36] R. He, S. Cao, J. Yu, Y. Yang, Microwave-assisted solvothermal synthesis of $\mathrm{Bi}_{4} \mathrm{O}_{5} \mathrm{I}_{2}$ hierarchical architectures with high photocatalytic performance, Catal. Today 264 (2016) 221-228.

[37] J. Li, H. Li, G. Zhan, L. Zhang, Solar water splitting and nitrogen fixation with layered bismuth oxyhalides, Acc. Chem. Res. 50 (2017) 112-121.

[38] J. Li, G. Zhan, Y. Yu, Superior visible light hydrogen evolution of Janus bilayer junctions via atomic-level charge flow steering, Nat. Commun. 7 (2016) 11480.

[39] J. Li, L. Cai, J. Shang, Y. Yu, L. Zhang, Giant enhancement of internal electric field boosting bulk charge separation for photocatalysis, Adv. Mater. 28 (2016) 4059-4064 (* The enhancement of the internal electric field in $\mathrm{Bi}_{3} \mathrm{O}_{4} \mathrm{Cl}$ was achieved by modulating its electronic structure through replacing $\mathrm{Cl}$ atoms with $\mathrm{C}$ atoms. The boosting bulk charge separation is then demonstrated by this strategy).

[40] C. Huang, C. Chen, M. Zhang, L. Lin, X. Ye, S. Lin, M. Antonietti, X. Wang, Carbon-doped BN nanosheets for metal-free photoredox catalysis, Nat. Commun. 6 (2015) 7698.

[41] Y. Qu, X. Zhong, Y. Li, L. Liao, Y. Huang, X. Duan, Photocatalytic properties of porous silicon nanowires, J. Mater. Chem. 20 (2010) 3590- 
3594.

[42] G. Liu, P. Niu, L. Yin, H.-M. Chen, $\alpha$-Sulfur crystals as a visible-light-active photocatalyst, J. Am. Chem. Soc. 134 (2012) 9070-9073.

[43] D. Xia, Z. Shen, G. Huang, W. Wang, J.C. Yu, P.K. Wong, Red phosphorus: An earth-abundant elemental photocatalyst for "green" bacterial inactivation under visible light, Environ. Sci. Technol. 49 (2015) $6264-6273$.

[44] M.Z. Rahman, C.W. Kwong, K. Davey, S.Z. Qiao, 2D phosphorene as a water splitting photocatalyst: Fundamentals to applications, Energy Environ. Sci. 9 (2016) 709-728.

[45] K. Ding, L. Wen, S. Huang, Y. Li, Y. Zhang, Y. Lu, Electronic properties of red and black phosphorous and their potential application as photocatalysts, RSC Adv. 6 (2016) 80872-80884.

[46] Z. Lou, B. Huang, Z. Wang, X. Ma, R. Zhang, X. Zhang, X. Qin, Y. Dai, M.-H. Whangbo, $\mathrm{Ag}_{6} \mathrm{Si}_{2} \mathrm{O}_{7}$ : A silicate photocatalyst for the visible region, Chem. Mater. 26 (2014) 3873-3875.

[47] A. Al-keisy, L. Ren, D. Cui, Z. Xu, X. Xu, X. Su, W. Hao, S.X. Dou, Y. Du, A ferroelectric photocatalyst $\mathrm{Ag}_{10} \mathrm{Si}_{4} \mathrm{O}_{13}$ with visible-light photooxidation properties. J. Mater. Chem. A 4 (2016) 10992-10999 (* Ag $_{10} \mathrm{Si}_{4} \mathrm{O}_{13}$ exhibited high visible-light photooxidation activity due to its efficient separation of photo-excited electon-hole pairs, which is contributed by the 
dispersive $\mathrm{CB}$ and flat $\mathrm{VB}$ ).

[48] X. Zhu, P. Wang, B. Huang, X. Ma, X. Qin, X. Zhang, Y. Dai, Synthesis of novel visible light response $\mathrm{Ag}_{10} \mathrm{Si}_{4} \mathrm{O}_{13}$ photocatalyst, Appl. Catal. B 195 (2016) 315-322.

[49] X. Zhu, Z. Wang, B. Huang, W. Wei, Y. Dai, X. Zhang, X. Qin, Synthesis of $\mathrm{Ag}_{9}\left(\mathrm{SiO}_{4}\right)_{2} \mathrm{NO}_{3}$ through a reactive flux method and its visible-light photocatalytic performances, APL Mater. 3 (2015) 104413.

[50] M. Li, Y. Dai, X. Ma, T. Jing, B. Huang, Insights into the effect of inner polarization and multiple Ag-O units on high-efficient Ag-based photocatalyst, Appl. Catal. B, 205 (2017) 211-218.

[51] Z. Lou, Z. Wang, B. Huang, Y. Dai, G. Wang, Z. Jiang, X. Zhang, X. Qin, Y. Li, One-step synthesis of amorphous silver silicates with tunable light absorption spectra and photocatalytic activities in the visible region, Chem. Eur. J. 21 (2015) 8706-8710 (* Amorphous silver silicates showed tunable light absorption spectra and photocatalytic activities in the visible region towards the modulation of the $\mathrm{Ag} / \mathrm{Si}$ ratio).

[52] T. Kako, Z. Zou, M. Katagiri, J. Ye, Decomposition of organic compounds over $\mathrm{NaBiO}_{3}$ under visible light irradiation, Chem. Mater. 19 (2007) 198202.

[53] Y. Ding, P. Zhou, H. Tang, Visible-light photocatalytic degradation of bisphenol $\mathrm{A}$ on $\mathrm{NaBiO}_{3}$ nanosheets in a wide $\mathrm{pH}$ range: a synergistic effect between photocatalytic oxidation and chemical oxidation, Chem. Eng. J. 
291 (2016) 149-160.

[54] W.-L. Zhou, Z.Y. Zhao, Electronic structures of efficient $\mathrm{MBiO}_{3}(\mathrm{M}=\mathrm{Li}$, Na, K, Ag) photocatalyst, Chin. Phys. B, 25 (2016) 037102. 


\section{Figures and figure captions}
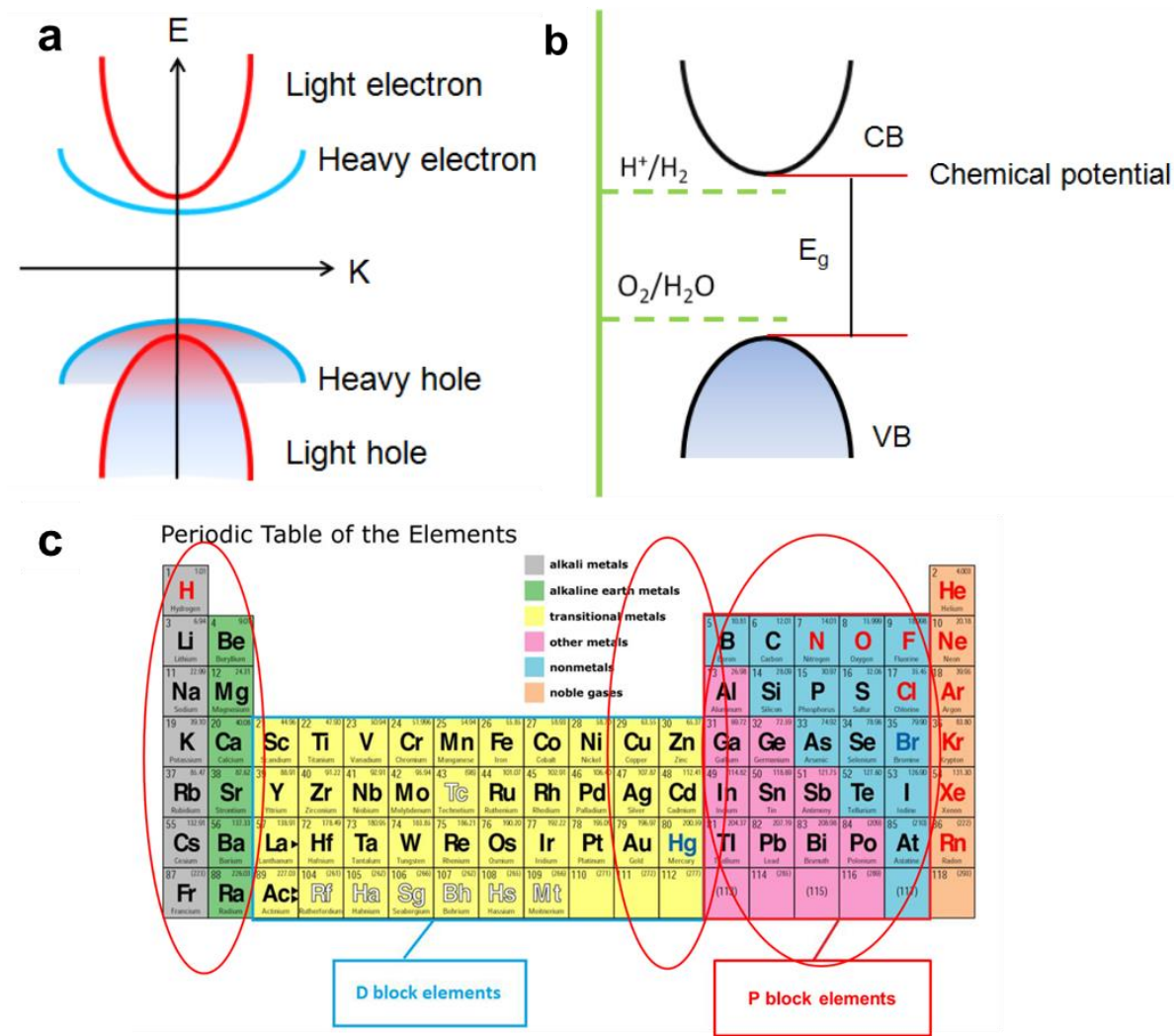

Fig. 1 (a) Diagram of the relationship between the band shape and the effective mass of electrons and holes. (b) Diagram of the band structure of direct-gap semiconductors, illustrating its role in determining the catalytic properties of photocatalytsts. (c) The periodic table of the elements with the IA and IIA, IB and IIB, and $p$-block regions marked using red ovals. 

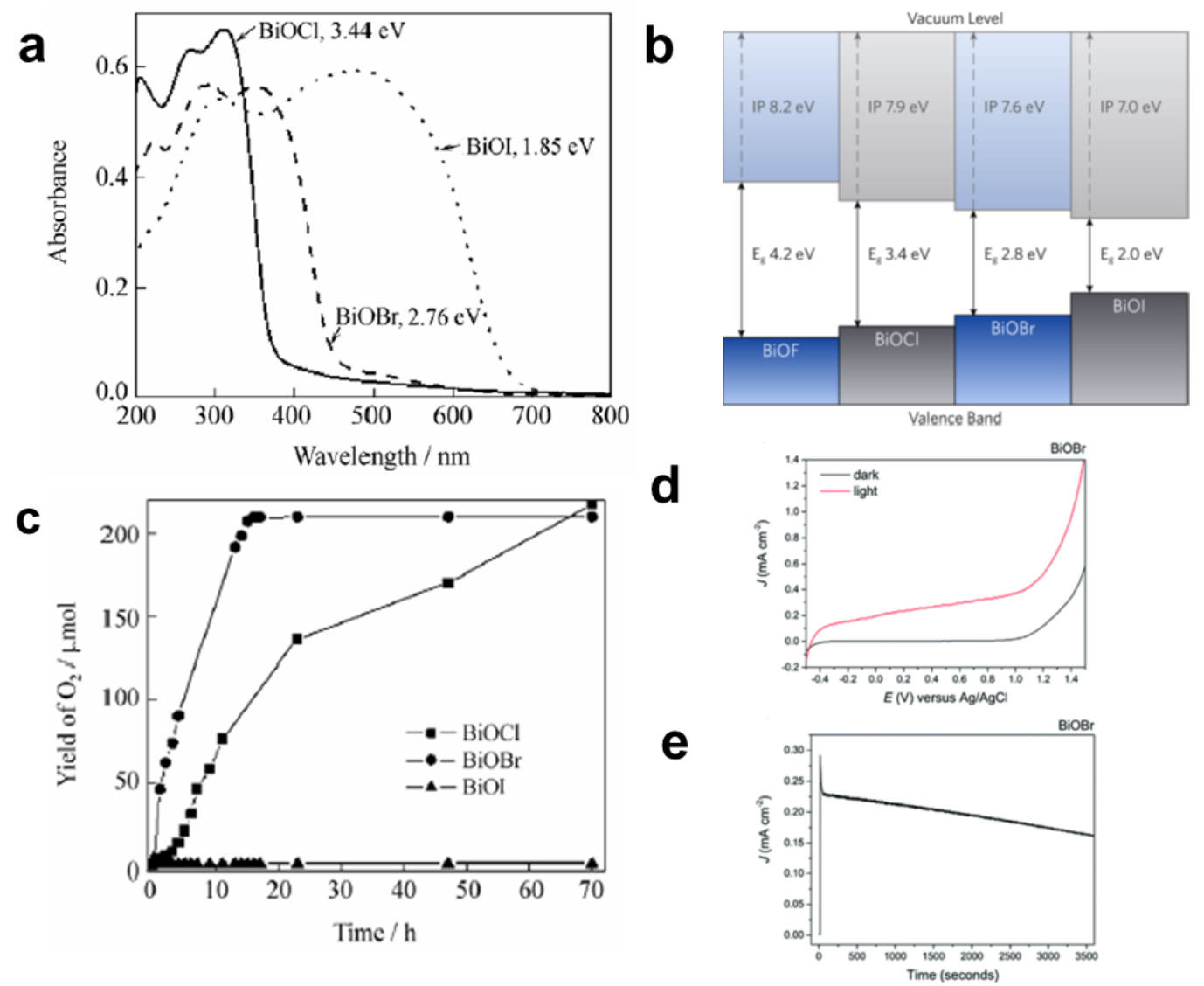

Fig. 2 (a) Ultraviolet-visible (UV-Vis) diffuse reflectance spectra of $\mathrm{BiOX}(\mathrm{X}=$ $\mathrm{Cl}, \mathrm{Br}$, and I) [29]. (b) Calculated band gap and the band alignment of the BiOX $(\mathrm{X}=\mathrm{Cl}, \mathrm{Br}$, I and F) compounds by density functional theory (DFT) [30]. (c) Oxygen evolution of BiOX under simulated sunlight irradiation [29]. (d) Photoanodic activity measurement of $\mathrm{BiOBr}$ film under simulated sunlight irradiation. (e) Stability of the BiOBr film at an applied voltage of $1.0 \mathrm{~V}$ vs. $\mathrm{Ag} / \mathrm{AgCl}[33]$. 


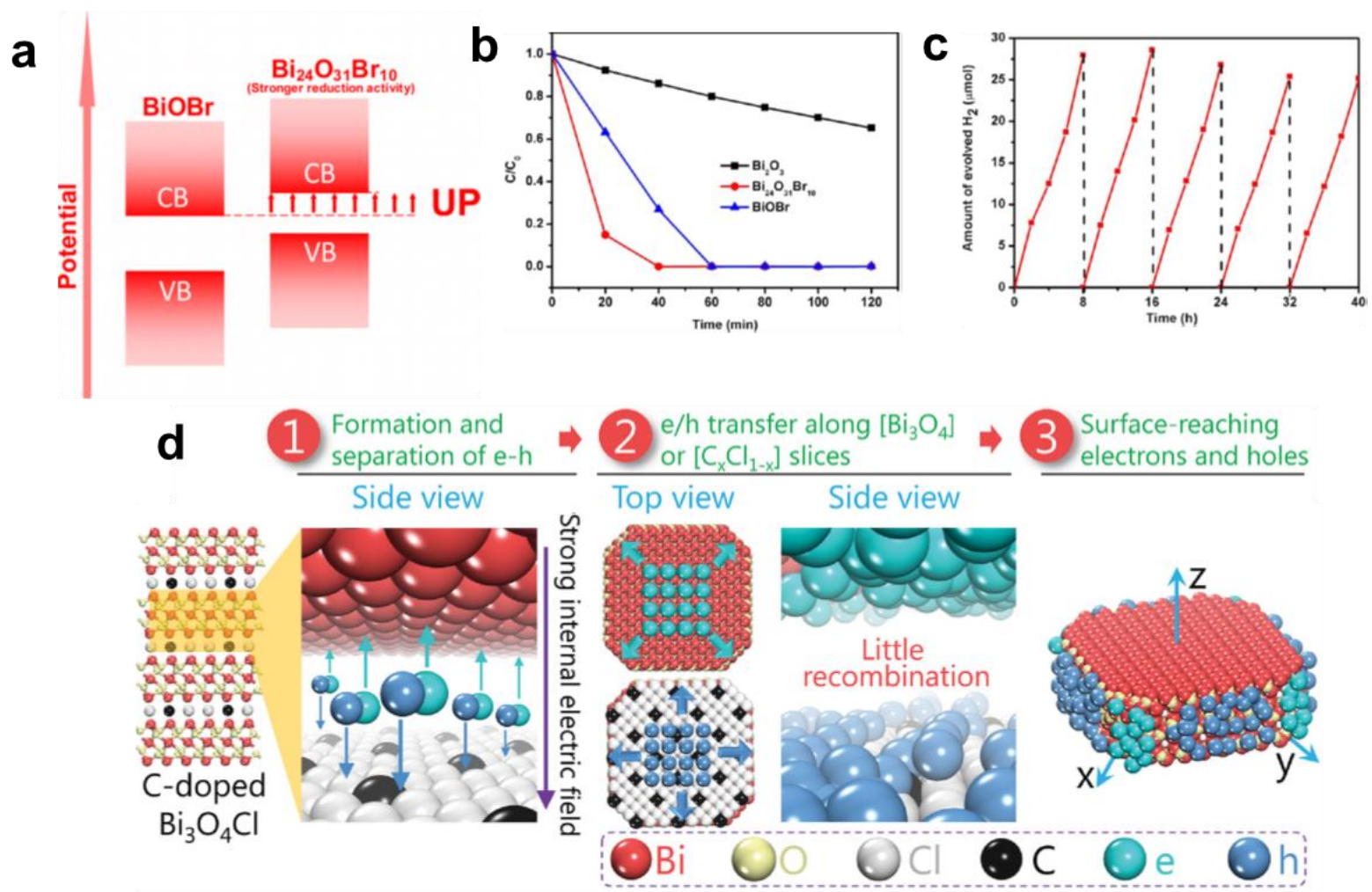

Fig. 3 (a) Schematic diagram of the uplifting of the CBM of $\mathrm{Bi}_{24} \mathrm{O}_{31} \mathrm{Br}_{10}$. (b) Experiments on photocatalytic reduction of $\mathrm{Cr}(\mathrm{IV})$ under visible-light irradiation by $\mathrm{Bi}_{24} \mathrm{O}_{31} \mathrm{Br}_{10}, \mathrm{BiOBr}$ and $\mathrm{Bi}_{2} \mathrm{O}_{3}$. (c) $\mathrm{H}_{2}$ evolution by water splitting using $\mathrm{Bi}_{24} \mathrm{O}_{31} \mathrm{Br}_{10}$ as photocatalyst under visible-light irradiation [6]. (d) Internal electric field (IEF)-mediated bulk-charge separation in carbon-doped $\mathrm{Bi}_{3} \mathrm{O}_{4} \mathrm{Cl}$ [39]. 

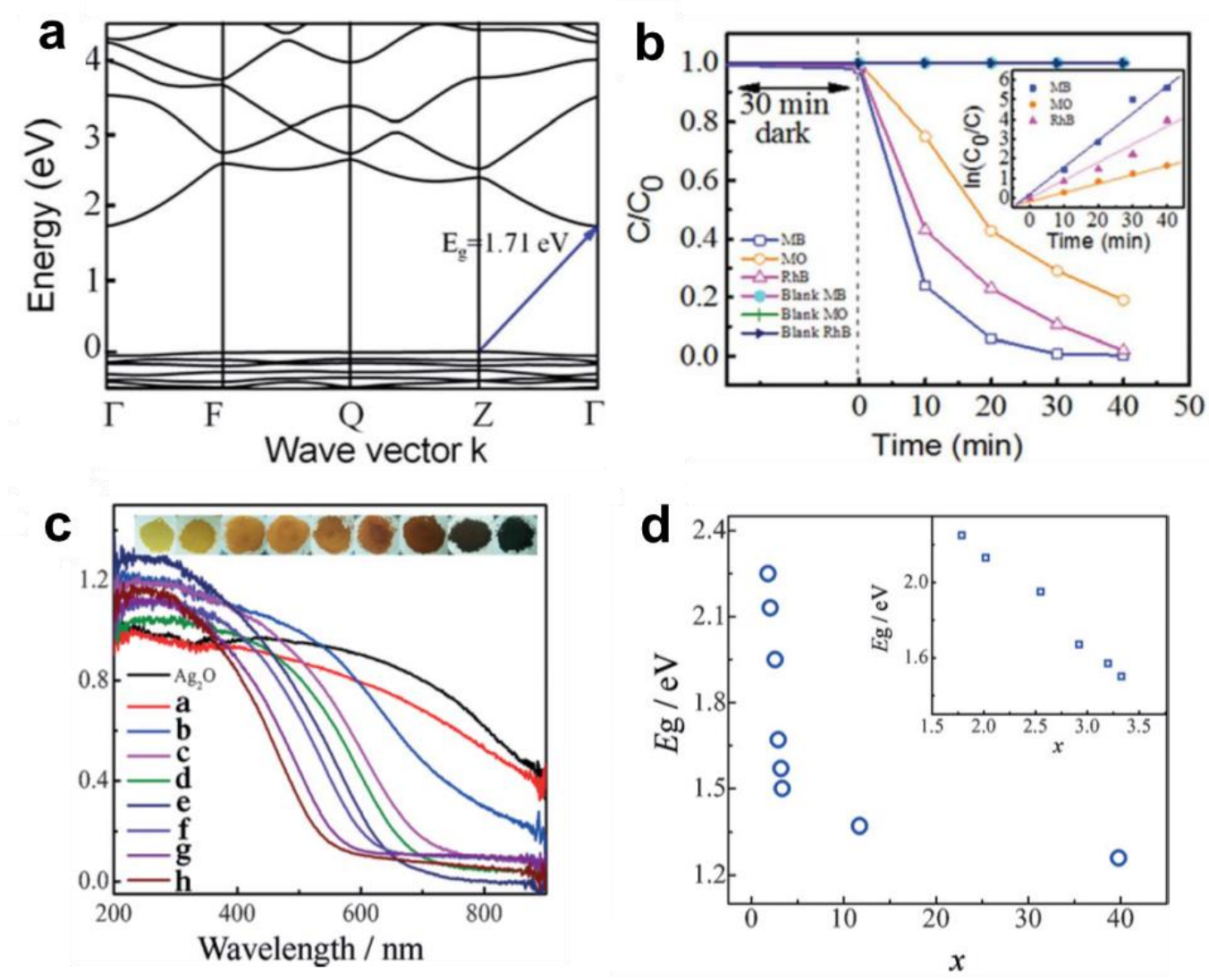

Fig. 4 (a) DFT calculated electronic structure of $\mathrm{Ag}_{10} \mathrm{Si}_{4} \mathrm{O}_{13}$, in which a dispersive $\mathrm{CB}$ and flat $\mathrm{VB}$ can be observed. (b) Photodegradation rate of organic dyes over $\mathrm{Ag}_{10} \mathrm{Si}_{4} \mathrm{O}_{13}$ under visible light; the inset shows the kinetics of the photocatalytic degradation process of organic dyes under visible light over $\mathrm{Ag}_{10} \mathrm{Si}_{4} \mathrm{O}_{13}$, indicating its high visible-light photocatalytic oxidation activity [47]. (c) UV-Vis diffuse reflectance spectra of $\mathrm{Ag}_{2} \mathrm{O}$ and silver silicates prepared with different $\mathrm{Ag} / \mathrm{Si}$ atomic ratios. (d) Estimated band gap $\left(E_{\mathrm{g}}\right)$ of amorphous silver silicates with different $\mathrm{Ag} / \mathrm{Si}$ atomic ratios $x$; the inset shows an enlargement of the indicated range [51]. 